\title{
XIII.
}

\section{Ueber hereditäre Nephritis und über den Hereditäts-Begriff im Allgemeinen.}

Von Dr. F. Samelsohn, Augenarzt in ${ }^{-}$Cöln.

Der Begriff der Heredităt spielt zur Stunde in der Aetiologie vieler Krankheiten eine so grosse Rolle, dass man sich füglich wundern darf, wie wenig die exacte Forschung bisher einer wissenschaftlichen Analyse desselben näher getreten ist. Denn in seiner wörtlichen Nacktheit, wie derselbe in der Pathologie zur Zeit besteht, hat dieser Begriff einer Entwickelung pathogenetischer Erkenntniss mebr geschadet, als genützt, wofür dio Geschichte der Krankheitswissenschaft Beweise in Fülle bieten dürfte. Vergleichen wir mit dieser Sterilität besagten Begriffes auf pathologischem Gebiete die frische. Triebkraft, mit welcher derselbe auf morphologischem und physiologischem Gebiete keimt und daselbst, wenn auch noch nicht neue Wabrheiten, so doch neue Bahnen der Fol'schung erobert hat, so dürfen wir den Moment nicht für verfrubt erachten, wenn wir die auf physiologischem Terrain adoptirte Methode auch auf unser palhologisches übertragen und fruchtbar zu erproben beginnen. Bisher begnügte man sich, unter den verschiedenen Bedingungen einer Krankheitsgenese die Erblichkeit zu verzeichnen; und selbst in den neuesten Handbüchern der Pathologie, deren Methode fast eine unbestritten exact-physiologische zn nennen ist, finden wir diesen Begriff noch in seiner ganzen nichtssagenden Allgemeinheit verzeichnet. So lange der Begriff der Krankheit ein einheitlicher, gleichsam individueller, war, so lange man die Krankheit als ein "Ens ontologicum" betrachtete, konnte man die Heredität als ein zweites "Ens" anstandslos in den Krankheits-Systemen führen. Heute aber, da wir die Krankheit als eine biologische Viel. heit, als eine einfache Abweichung und Veränderung des normalen, physiologischen Prozesses ansehen, müssen wir auch den alten, einheitlichen Begriff der Heredität fallen lassen und ihn in seine vielfachen Elemente zerlegen, durch deren ges ondertes Studium wir 
vielleicht $\mathrm{zu}$ einer Erkenntniss seiner Bedeutung in physiologischen und pathologischen Verhältnissen gelangen werden. Es muss neben der Thatsache, ob gewisse Krankheiten sich vererben, auch der Modus untersucht werden, in welcher Weise sie sich vererben, ob das Geschlecht von Eltern und Kindern, welche eine gewisse Krankheit durch Vererbung gemeinsam besitzen, in einem bestimmten, und in welchem Verhältnisse stehe; in welchem Lebensalter ein Kind von der ererbten Krankheit betroffen werde; welche Einzelelemente der Krankheit durch Vererbung übertragen werden, welche nicht, und welcbe also nur eine Entwickelung der übertragenen repräsentiren, welche derselben durch Vererbung sich cumuliren, welche sich abstumpfen: kurz es müssen alle die Punkte einer unbefangenen statistischen Untersuchung unterzogen werden, durch deren Analyse auf morphologischem Gebiete Darwin zu seiner Theorie der Pangenesis gelangt ist ${ }^{1}$ ).

Wenn ich nun für eine Krankheitsform, für welche ein Erblichkeitsverhältniss bisher meines Wissens noch nicht erwähnt worden ist, ein solches durch eine Beobachtung zu constatiren versuchen will, so dürfte aus obigen Auseinandersetzungen leicht folgen, dass eine solche Gabe für die Wissenschaft eine sehr zweifelhafte sei, da sie den unfruchtbaren Wust des alten Erblichkeitsbegriffes nutzlos vermehren hilft. Aber es kommt ja zunächst darauf an, für gewisse Erkrankungen die Thatsache der Heredität nackt zu constatiren; und wenn ich im Stande bin, dieser nackten Thatsache noch einzelne Details hinzuzufügen, welche die postulirte Methode illustriren können, so halte ich die Publication einer derartigen Beobachtung für hinreichend gerechtfertigt.

Sebastian B., ein grosser, kräftiger Mann ron 59 Jahren, consultirt mich am 21. November 1871 wegen einer geringen Abnahme seiner Sehschärfe, "die iln zwar nicht in seinen Beschältigungen störe, jedoch durch den ab und zu auftretenden Nebel vor seinen Augen genire." Bei der Untersuchung seiner Sehschärfe finde ich, nach Correction einer geringen Hyperopie, dieselbe fast normal: das geringe Deficit, das sich kaum durch einen exacten Bruch ausdrücken lässt, erkläre ich durch sein vorgeschrittenes Alter, in welchem bekanntlich, ganz in der Breite

1) Darwin, Das Variiren der Thiere und Pflanzen im Zustande der Domestication. Deutsch von Victor Carus. Bd. 2. S. 99. Abstammung des Menschen und die geschlechtliche Zuchtwahl. Bd. I. S. 248. Vergl. auch Hering, Ueber das Gedächtniss als eine allgemeine Function der organischen Materie. Vortrag am 30. Mai 1870 in der Wiener Akademie. 
des Normalen, Bedingungen für eine geringe Herabsetzung der Sehschärfe liegen können. Bei der ophthalmoskopischen Untersuchang ergiebt sich eine ganz minimale äquatoriale Trübung der rechten Linse, die übrigen Medien jedoch völlig durchsichtig. Beide Netzhäute zeigen zablreiche Apoplexien, die flecken- und streifenförmig besonders den 0pticus-Eintrilt umgebea, die Papille selbst aber freilassen. Eine geringe circumpapilläre Trübung, die die Contouren der Papille aber nicht verhüllt, etwas enge Arterien, dagegen erweiterte und geschlängelte Venen setzen das bekannte Bild der Retinitis apoplectica zusammen, wie es von Vielen als das erste Stadium der specifischen Retinitis nephritica geschildert wird 1). Eine auf die supponirte Nierenerkrankung zielende Organuntersuchung ergiebt alle Symptome einer diffusen Nephritis am Ende des zweiten Stadiums: heller, etwas vermehrter Urin, nackte und mit körnigem Detritus bedeckte Fibrincylinder, enge und gespannte Arterien, exquisite Vergrösserung des linken Ventrikels, geringer Bronchialkatarrh; beine 0edeme, ungestörte Verdauung. Nar vor Jahresfrist giebt er an, ohne jede Veranlassung Blutaustretungen an beiden unteren Augenlidern wahrgenommen zu haben, die sich im Laufe einiger Wochen wieder von selbst resorbirten.

Als ich den Patienten dann weiter auf die Symptome des Nierenleidens inquirirte, gab er mir an, dass "das Wasser" in seiner Familie häufig rorkomme, dass bereits 2wei Schwestern und ein Bruder am Wasser gestorben seien, und dass auch der eine Bruder einige Zeit vor seinem Tode an den Augen erkrankt und von mir behandelt worden sei. Ich erinnerte mich bald, einen Kranken desselben Namens vor etwa 3 Jahren an einer Retinitis apoplectica behandelt zu haben, worüber mir mein Krankenjournal folgenden Aufschluss giebt:

Peter B., 59 Jahre alt, consultirte mich am 11. Juli 1869 wegen einer weit vorgescbrittenen Amblyopie: beiderseits nur quantitative Lichtempfindung bei normaler Projection. Links Papille weiss, rechts blasser als normal, Arterien selir eng, Venen verbreitert und geschlängelt. Beiderseits Extravasate um die Papille. Eiweiss und Formelemente im trin, Hypertrophie des linken Ventrikels.

Ich finde sodann eine zweite Notiz vom 12. October 1869, welche besagt, dass die Atroplie der 0ptici vorgeschritten ist, und sich beiderseits Extravasate a uf der Papille zeigen. Sodann ist eine Erhöhung des intraoculären Druckes verzeichnet ohne Pulsation der Arterien; endlich Oedem der Füsse und dyspnoëtische Beschwerden. Der Tod trat im December desselben Jahres unter den Erscheinungen einer Apoplexie ein, wovon mir aus dem Heimathsorte des Patienten damals Mittheilung gemacht worden war. Auch Sebastian B. ist mittlerweile im Mai dieses Jahres unter den Erscheinungen der Urämie gestorben, ohne dass die Retinitis apoplectica sich zu der specifischen Retinitis nephritica entwickelt bätte: vielmehr war bis zu seinem Tode die Sehschärfe eine befriedigende, und auch die Oedeme stellten sich nur in mässigem Grade ein, sodass seine Beschwerden sich besonders auf Kopf and Athmung bezogen. Eine Section konate leider nicht gemacht werden.

1) Rosenstein; Nierenkrankheiten. S. 180. 
Diese beiden Fälle, so fragmentarisch auch die Beobachtung des Peter B. gewesen ist, werfen doch eine eigenthümliche Beleuchtung auf einander, zusammengehalten mit den Todesursachen zweier Schwestern, die gleichfalls in dem Alter von 55 bis 60 Jahren "am Wasser" ohne Augenerk rankung gestorben sind. Ueber die Eltern konnte ich nur erfahren, dass der Vater an einer Verletzung, die Mutter jedoch am Schlage gestorben sei.

Betrachten wir nehmlich die fraglichen Fälle vorurtheilsfrei, so finden wir bei 2 Bridern constatirte diffuse Nephritis mit HerzHypertrophie und Nétzhaut-Extravasaten, bei 2 Schwestern wahrscheinliche Nephritis (wiewohl eine andere Ererbung des Anasarca nicht ausgeschlossen ist), bei der Mutter Apoplexie, als deren Ursache Morbus Brightii wenigstens nicht ausgeschlossen ist. Es dürte desshalb die Annahme einer hereditären Anlage zn dieser Erkrankung nicht zurủckzuweisen scin, da ein Zufall, der sämmtliche Geschwister einer Nierenerkrankung unterwirft, einer bereditären Ursache gleichkommt. Diese nackte Disposition gewinnt 'ein erhöhtes Interesse durch die Altersstufe, in welcher sämmtliche erwähnte Individuen mit einer aufallenden Gleichartigkeit von dem Uebel betroffen wurden: 4 Geschwister sind an der Grenze des 60. Jahres gestorben. Wonn dieses Alter nun auch nicht sehr fern von dem durch Bibel und Erfahrung für einen normalen Organismus festgesetzten Extinctions-Termine steht, so lässt doch die gleichmässige Todesursache wenigstens bei den beiden von mir beobachteten kräftigen Männern, bei denen von Alters-Atrophie nach keiner Rientung die Rede sein konnte, daran denken, ob nicht ein von dem mutterlichen Organismus überkommener Krankheitskeim einer Entwickelung von einer ganz bestimmten Dauer bedürfe, um als Krankheit in dem Sprösslinge in die Erscheinung zu treten, ganz ähnlich wie gewisse Keime unter physiologischen Bedingungen einer bestimmten Zeit bedürfen, um als Zühne, als Bartund Schaamhaare, als Samenfäden, Eier etc. zu erscheinen.

Dürfte die obige Mittheilung Material bieten für die B̌ehandlung der beiden Fragen, ob sich die Anlage zur diffusen Nephritis vererben könne, und ob diese Anlage in einer bestimmten Lebensepoche zum Ausbruche gelangen müsse, so lässt sie sich noch von einem anderen Gesichtspunkte für die Entwickelung des Hereditätsbegriffes fruchtbar verwerthen, nehmlich zur Analyse der Frage, 
a uf welche Weise, d. h. durch die Vererbung welcher Krankheitselemente eine solche Disposition übertragen werden könne. Es benöthigt hierzu einer genaueren Analyse des fraglichen Krankheitsbildes. Wir finden bei beiden Brüdern Sehstörungen verzeichnet mit der objectiven Grundlage von Retinitis apoplectica, Erkrankungen, die bei den beiden Schwestern gefeblt zu baben scheinen, wenigstens nicht verzeichnet sind, wiewohl man sich erinnern mag, dass bedeutende Veränderungen in der Retina ganz besonders bei Nephritis beobachtet werden, ohne dass denselben eine proportionale Herabsetzung der Sehschärfe entspräche. Ohne mich in vage Hypothesen über den Einfluss des Geschlechtes auf die Vererbung einzulassen, für dessen Bedeutung die obige Beobachtung nicht einmal schätzbares Material zu nennen ist, wollen wir hier vielmehr die Frage discutiren, welche Bedeutung die Retinitis apoplectica in dem Krankheitsbilde der diffusen Nephritis habe. Ich bemerkte bereits vorher, dass die Retinitis apoplectica als das erste Stadium der specifischen Retinitis nephritica von Vielen angesehen werde, aus welchem Umstande dieselben ein Abhängigkeitsverhältniss zwischen der Netzhauterkrankung und den allgemeinen Circulationsstörungen deduciren zu dürfen glauben ${ }^{1}$ ). Eine unbefangene Betrachtung zeigt jedoch, dass ein solches Abhängigkeitsverhältniss in so einfacher Weise, wie es z. B. Schweigger mit Heranziehung der Hypertrophie des linken Ventrikels annimmt, nicht besteht, ebensowenig wie ein nothwendiger Zusammenhang $\mathrm{z}$ wischen Retinitis apoplectica und specifischer Retinitis nephritica. Denn wenn auch jede specifische Retinitis nephritica mit den Erscheinungen der Netzhauthyperämie und ihren begleitenden Symptomen beginnen dürfte, welches Bild in seiner Entwickelung von Liebreich ${ }^{2}$ ) so treffend geschildert worden, so ist damit doch nicht bewiesen, dass eine solche Hyperämie der Nelzhaut bei Morbus Brightii za den Folgeerscheinungen der Verfettung des Bindegewebes und der Sklerose der nervösen Elemente führen müsse, wre sie für die Retinitis nephritica charakteristisch sind. Und in der That zeigt der Fall des älteren Bruders, dass bereits der grösste Theil des Sehvermögens geschwunden, dass weit vorgeschrittene Atrophie der

1) Rosenstein l. c.; Wecker, Maladies des yeux. II. p. 342; Seitz und Zehender, Handbuch d. Augenheilk. II. S. 589-591.

2) Archiv für Ophthalmologie. V, 2, 
Nervi optici eingetreten sein könne, ohne dass auch nur eine Spur der charakteristischen Veränderungen der nervösen und bindegewebigen Netzhautelemente zu der einfachen Gefässerkrankung sich geselit hätte, deren objectives Zeichen die Extravasate sind. Auch in dem Falle des jüngeren Bruders, der bis zum Tode unter Beobachtung blieb, ist gleichfalls nur Gefässberstung zu constatiren, wiewohl das Nierenleiden schon beim Eintritte der ersten Sehstörungen so weit vorgeschritten war, um einer Fortentwickelung des Netzhautleidens von dieser Richtung her jeden erdenklichen Vorschub zu leisten. Es folgt aus dieser Gegenüberstellung, dass, wenn wir Fälle finden, in denen auf der Basis einer Nierenerkrankung Nelzhautextravasate ohne darauf folgende specifische Netzhautentartung bis an's Lebensende bestehen, ein genetisches Abhängigkeitsverbältniss zwischen diesen beiden Erkrankungen der Netzhaut nicht bestehen $\mathrm{m} u ̈ \mathrm{sse}$, welche Ansicht bereits $\mathrm{Nagel}$ ungeschminkt ausgesprochen hat ${ }^{1}$ ).

Sehen wir also als das bei den Netzhauterkrankungsformen gemeinsame Symptom die Apoplexien, oder vielmehr die Extravasate auftreten, so werden wir zunächs deren Entstehungsgeschichte zu studiren haben. Eine auch nur oberflächliche Hindeutung auf den Mechanismus der intraoculären Circulation beweist uns, wie wenig günstig derselbe der Entstebung von Netzhautextravasaten nur in Folge der Erhöhung des Druckes in der Aorta ist. Denn wenn schon die einfache Thatsache, dass Netzhautextravasate bei Hypertrophie des linken Ventrikels in Folge von Klappenfehlern nicht zu den häufigen Befunden gehören, schlecht mit einer solchen Annahme tubereinstimmt, so ist andererseits die gegenseitige Regulirung zwischen äusserem Blut- und innerem Augenflüssigkeitsdrucke in der geschlossenen Bulbuskapsel eine so feine, dass, wie bekannt, selbst das arterielle Pulsphänomen in den intraoculären Gefässen schwindet. Wir sehen also, dass einseitige Erhöhung des Blutdruckes in der Aorta, dessen plötzliche Schwankungen unter ganz physiologischen Verhältnissen nicht überaus selten sind, die Circulationsverhältnisse in der Netzhaut ebensowenig bis zur Zerreissung der gesunden Gefässwände zu alteriren vermag, wie z. B.

1) Die fettige Degeneration der Netzhaut, Archiv f, Ophthalm. 1860. Bd. VI, 1. S. 233 , 
die gesunden Gefässe der geschlossenen Schädelkapsel, deren Circulationsverhältnisse mit denen im Auge grosse Analogien bieten. Anders jedoch gestalten sich hier wie dort die Verbältnisse, wenn die Gefässwände krankhaft entartet sind, und so der erhöhten Pulswelle nicht mehr den genügenden Widerstand entgegenzusetzen vermögen. Die anatomischen Untersuchungen in Fällen specifischer Retinitis nephritica ergaben nun ausreichende Veränderungen der Gefässwände, um ihre verminderte Widerstandsfähigkeit zu begreifen: circumscripte Verfettung, theilweise Sklerose, Hypertrophie der Adventitia sind die Veränderungen, die den ausgesprochenen Fällen der charakteristischen Retinitis selten fehlen. Eine ähnliche Veränderung der Gefässwände werden wir nach den vorhergehenden Auseinandersetzungen für diejenigen Fälle supponiren dürfen, in denen die gesammten sichtbaren Veränderungen der Netzhaut in zablreichen Extravasaten bestehen; eine Annahme allerdings', für welche der anatomische Beweis noch zu erbringen ist.

Die beiden Brudern gemeinsame Netzhautveränderung, zahlreiche Extravasate in der Netzhaut, weist uns somit direct auf das Gefässsystem, für dessen krankhafte Alteration eine gemeinsame ererbte Disposition vorzuliegen scheint. Gilt diese Disposition allerdings zunächst nur für die Netzhautgefässe, und bei Sebastian vielleicht für die Palpebral - und Conjunctivalgefässe, so werden wir bei der weiteren Gemeinsamkeit der allgemeinen Erkrankung wenigstens den Versuch wagen dürfen, einen Zusammenhang in das gesammte Krankheitsbild zu bringen.

Es ist bekanntlich in jügster Zeit mit Erfolg der Versuch gemacht worden, die angeborne abnorme Beschaffenheil des Gefässsystems in eine ätiologische Beziebung zu der Entwickelung von Krankheiten zu bringen. Beneke ${ }^{1}$ ) gab, zuerst in der Gesellschaft zur Beförderung der gesammten Naturwissenschaften zu Marburg 1868, das Resultat von 96 Messungen der Gefässlumina und deutete auf Grund derselben die Möglichkeit an, dass die normwidrige Enge der Gefässlumina, mit der er sehr häufig abnorm weite Oeffnungen der Arteriae coronarie cordis vergesellschaftet fand, in ätiologischem Zusammenhange stehen könne zu gewissen Allgemeinerkrankungen, als welche er besonders Scrophulose mit nephriti-

1) Jahrbuch für Kinderheilkunde u. physische Erziehung. IV, 4. 1871. S. 386-389. 
schen Prozessen und amyloider Gefïssentartung nennt. Eine gleiche ätiologische Idee versucht Virchow ${ }^{1}$ ) für die Chlorose zu erweisen und stütat dieselbe mit anatomischem Material und physiologischen Deductionen. Er weist nach, wie aus der angebornen Enge des Aortensystems, der in der fribesten Zeit des Lebens. Kleinheit des Herzens entsprechen mag, in späteren Zeiten des Wachsthums sich Hypertrophie des Herzens entwickelt, wie aus der angebornen Dünnheit und Zartheit solcher engen Gefässwände (Rokitansky), verbunden mit deren bekannter Prädisposition zu Erkrankungen (Fettmetamorphose der Intima, Media und des Herzfleisches), sich die Neigung zu Gefässrupturen erklärt, und wie endlich aus solchen angebornen Gefässanomalien secundär manche Erkrankungen der Parenchyme und besonders der Nieren entstehen können. „Die grosse Häufigkeit, mit der gerade die Nierenarterien gleichzeitig init den Erkrankungen des Parenchyms schwere Veränderungen ihrer Wandungen erfahren, ist bekannt, und wenn man die Fälle von Herzhypertrophie mit Granularatrophie der Nieren prüft, so liegt es gewiss nahe, diejenigen hierher zu ziehen, in denen sich zugleich eine mangelhafte Entwickelung der Aorta nachweisen lässt" ${ }^{2}$ ). [Eine solche Möglichkeit der Entstehung von Nepbritis aus Erkrankung der grösseren Gefässe spricht bereits Rosenstein ${ }^{3}$ ) aus, indem er jhr Zustandekommen nicht auf entzündliche Vorgänge, sondern auf mangelnde Ernährung, ähnlich der senilen Atrophie, zurückführt.]

Zur Stütze dieser seiner Vermutbung fuhrt Virchow 2 Sectionsresultate an, in denen Enge der Aorta mit figurirter Fettentartung der Intima im Verein mit Granularatrophie der Nieren und Hypertrophie des Herzens nachgewiesen werden konnte; und von der einmal auf diesen Punkt gelenkten Aufmerksamkeit der Anatomen haben wir hoffentlich bald ein reicheres Material zu erwarten. Hierher müssen wir auch eine Beobachtung von George Johnson ${ }^{4}$ ) zählen, welcher bei Bright'scher Krankheit bedeutende Hyper-

1) Ueber die Chlorose und die damit zusammenhängenden Anomalien im Gefässapparate. 1872. (Nach einem Vortrage in der Berl. Geburtshülfl. Gesellschaft am 12. Juli 1870.)

2) Chlorose S. 26.

3) Nierenkrankbeiten 1863. S. 202.

4) The proximate cause of haemorrhage into the brain and retina in cases of chronic Bright's disease. Med, Times and Gaz. Vol. 41. p. 3-4. 
trophie der Wände der kleinen Arterien, sowohl in den Nieren, als in den verschiedensten Körpertheilen, fand. Der vermehrte Widerstand in diesen verengten Gefässen setzt Hypertrophie des Herzens, und in Folge dessen ganz besonders erhöhten Druck und Gefahr der Berstung in solchen Gefässen, deren Wände nicht verdickt sind. Als solche fand er in einem Falle von Hirnhämorrhagie die Gefässe der Pia mater. In dieser Beobachtung ist für uns von Interesse die weit verbreitete Verengerung der Gefässvolumina, welche Johnson allerdings auf die aurch die Nierenkrankheit veränderte Beschaffenheit des Blutes zurückführt, welche aber ohne besondere Vergewaltigung der Thatsachen eine Deutung in unserm Sinne zulassen dürfte. Auch Frerichs ${ }^{1}$ ) spricht bereits die Meinung aus, dass die Herzhypertrophie der Nierenerkrankung meist vorangehe und zum causalen Momente für letztere werde.

Durch eine solche Entwickelung des Krankheitsbildes - angeborne Enge des Gefässsystemes mit vermehrter Prädisposition desselben zu Erkrankungen seiner Wände, Hypertrophie des Herzens, Parenchymerkrankung der Niere - ebenen wir uns die Schwierigkeit der Deutung der Herzhypertrophie, deren mechanische Erklärung bei der bisherigen Construction des betreffenden pathologischen Bildes schlechterdings nicht gelingen wollte. Nur eine mangeihafte Uebertragung klayer hämodynamischer Principien auf pathologische Verhältnisse konnte eine Zeit lang die Annahme bestehen lassen, als ob der Ausfall eines Capillargebietes, wie er bei der schrumpfenden Granularatrophie am Ende eintreten muss, eines Capillargebietes, das aber selbst in den vorgeschrittensten Stadien immer nur ein verhältnissmässig kleines Stromgebiet des Körpers betrifit, den Druck im Aortengebiete derartig zu erhöhen vermag, dass dadurch eine Hypertrophie des linken Ventrikels entstehen könne. $\mathrm{Zn}$ den zablreichen Bedenken, welche sich eincr solchen Annahme a priori entgegenstellen, und welche in der Literatur einen hinreichenden Ausdruck gefunden haben, gesellt sich neuerdings auch der experimentelle Gegenbeweis, wie er von Rosenstein ${ }^{2}$ ) angebahnt worden ist. Derselbe fand nach Exstirpation einer Niere, mit welcher ein Ausschluss des betreffenden capillaren

1) Die Bright'sche Nierenkrankheit. -1851 .

2) Ueber complementăre Hypertrophie der Niere, Dieses Archiv 1871, LIII, 2 u, 3. S. 154 . 
Stromgebietes eo ipso erreicht ist, keine merkliche Erhöhung des Aortendruckes und scbliesst hieraus, dass auch bei der diffusen Nephritis das wesentliche Moment für den gesteigerten Aortendruck und der daraus resultirenden Herzhypertrophie nur indirect in der Verödung eines Theiles der Capillaren zu suchen ist, insofern dadurch die Wasserausscheidung des Blutes beträchtlich verringert wird, und möglicherweise auch insofern ein abnormer Bestandtheil desselben reizend einwirkt. Mögen wir dieser letzteren Erklärungsweise zustimmen oder nicht, immer werden wir dieselbe doch nur auf diejenigen Fälle anwenden können; wo sich die Herzhypertrophie zu den Endstadien der Nephritis diffusa gesellt, während sie auf jene ebenso zahlreichen kein Licht wirft, in denen die Herzhypertrophie sicher schon bei dem ersten Stadium der Nierenerkrankung zu constatiren ist, wo nicht allein keine Verringerung der Wasserausscheidung, sondern vielmehr das Gegentheil gefunden wird, wie nabe der Gedanke liegl, dass in diesen Fällen die Herzbypertrophie der Nierenkrankheit bereits vorangegangen sei, haben wir bereits ausgeführt.

Es bleibt also immer noch eine rein mechanisebe Erklärung der Hypertrophie des linken Ventrikels zu geben. Und eine solche erscheint ganz analog der Entstehung derselben bei Chlorose auf der Basis einer allgemein verbreiteten Verengung des Stromgebietes bei relativ zu grosser Blutmasse, die zugleich eine bessere Ernährung des Herzfleisches voraussetzt. ${ }^{1}$ ) Denn nur bei dieser Voraussetzung, dass die Vermehrung des Blutes mit der Entwickelung des Organismus unabhängig von der angebornen Verengerung der Gefässbahn vor sich geht, treffen die Relationen zwischen Herzarbeit und Circulationswiderständen zusammen, wie sie für die mechanische Erklärung der Herzhypertrophie erheischt werden. Es sind diese Verhältnisse theoretisch klar entwickelt worden in einer Dissertation von Fr. Fuchs ${ }^{2}$ ), und experimentell von Blasius, der nash der Methode von Ludwig am ausgeschnittenen Froschberzen, das er in einen künstlichen Kreislauf einschaltete, „Versuche über die Herzarbeit unter verschiedenen innerhalb des

1) Virchow, Chlorose. S. 10.

2) Veber die mechanischen Bedingungen der Hypertrophie und Dilatation des Herzens. Bonn 186\%. 
Kreislaufes herrschenden Druckverhältnissen " anstellt. ${ }^{1}$ ) Das von ihm gefundene erste Gesetz, das uns für unsere Frage am meisten interessirt, lautet:

„Das Maximum der Arbeitsleistung eines Froschherzens tritt bei um so höheren Werthen des arteriellen Druckes ein, ein je höherer Füllungsdruck angewandt wird. "

Und da ,das Herz als Muskelapparat in seiner Entwickelung von seiner Arbeitsleistung abhängig ist", so wird es klar, wie ein Wachsen der Blutmenge unabhängig von der Entwickelung der Gefässbahnen, welches Wachsen der physiologische Ausdruck des experimentellen Füllungsdruckes ist, durch vermehrte Arbeitsleistung zur Hypertrophie führen muss. Der anatomische Ausdruck dieser vermehrten Arbeitsleistung würde für gewisse Fälle in der oben citirten Beobachtung von Beneke liegen, in welcher bei allgemein verbreiteter Verengerung der Gefässlumina relativ we ite Oeffnungen der Arteriae coronariae gefunden wurden.

Würden wir, gestützt auf diese Aunahme - angeborne oder erworbene Enge eines grösseren Abschnittes des arteriellen Gefässsystems -, eine mechanische Erhlärung für die Entstehung der Herzhypertrophie und Gefässzerreissungen in Hirn - und Netzhaut in befriedigendem Maasse gewinnen, so würden wir zugleich zu einer mit den klinischen Thatsachen durchaus übereinstimmenden ätiologischen Scheidung des klinischen Begriffes "diffuse Nephritis“ gelangen. Denn wenn die anatomische Thatsache der Nierenschrumpfung auf der einen Seite nur in einer gewissen Quote aller Fälle mit Herzhypertrophie verbunden ist, auf der anderen Seite aber Herzhypertrophie nicht selten bereits in den Anfangsstadien der diffusen Nephritis gefunden wird, so ist es wohl klar, dass ein a llgemeineres pathogenetisches Erklärungsprincip herangezogen werden muss, als es in der Verödung eines kleinen Capillargebietes mit seinen mechanischen Consequenzen liegen dürfte: Leider aber besitzen wir noch nicht hinreichendes anatomisches Material, um schon jetzt mindestens zwei differente Formen der Nierenerkrankung pathologisch von einander zu sondern, die eine auf der Basis einer Gefässdegeneration mit consecutiver Parenchymerkrankung, die andere einer primären Parenchymerkrankung mit consecutiver Alteration

1) Würz̧burger Verhandlungen 1871 . Bd. II. Hft. 1 u. 2 . 
der Gefässe und Säfte: die erste Reihe würde mit Prägnanz diejenigen Störungen im Gefolge haben, welche uns als rein mechanisch-circulatorische imponiren (Herzhypertrophie, Hirn- und Netzhautextravasate), die zweite diejenigen, für welche wir eine mehr chemisch-nutritive supponiren (Retinitis nephritica mit der specifischen Degeneration der Netzhautelemente, Urämie?). So würden wir auch den Modus begreifen können, durch welchen sich nicht allein eine Disposition zur Nierenkrankheit, sondern auch deren Ausbruch in einer bestimmten Frist vererben könne: der Grad der Enge des Gefässsystems' und der krankhaften Alteration seiner Wandungen bedarf einer gewissen Zeit, um denjenigen Complex von circulatorischen und nutritiven Störungen zur Entwickelung zu bringen, der schliesslich das Krankheitsbild der Nephritis darstellt.

Ich bin mir wohl bewusst, dass dieses ganze Raisonnement, zu welchem mir eine detaillirtere Analyse der erwähnten Beobachtungen Veranlassung gegeben hat, zur Zeit nur hypotbetisch genannt werden muss, dem als reale Basis vielleicht nur die beiden erwähnten Sectionsbefunde Virchow's dienen dürften. Aber gerade diese Sectionsbefunde lassen mich hoffen, dass ein bewusstes Suchen nach den angedeuteten Abnormitäten des Gefässsystems in gleichem Maasse ergiebiger sich gestalten werde, in welchem wir lernen werden, die geringeren Abweichungen in der Weite der Gefässbahnen von der noch nicht genügend gekannten mittleren Norm zu erkennen und $\mathrm{zu}$ messen. Immerhin zeigt eine solche Hypothese, wie wir den ätiologischen Hereditätsbegriff aus seiner unfruchtbaren Allgemeinheit zu einem verständlichen morphologischen und physiologischen Agens einengen können.

Eine Krankbeit, welche bei ihrer so scharf ausgesprochenen erblichen Disposition die Versuchung zu ähnlichen analytischen Raisonnements nahe legt, ist die Myopie und deren Verhältniss zur Sklerochorioiditis posterior. Man findet nicht selten Fälle, in denen bei constatirter Kurzsichtigkeit eines elterlichen Organismus mit Sklerochorioiditis posterior sich bei einer gewissen Quote der Kinder Myopie mit Sklerochorioiditis, bei der anderen ohne dieselbe, findet, und bei der letzteren trotz eminenten progressiven Charakters der Myopie uncomplicirt bleibt, während bei der ersteren die Sklerochorioiditis erbebliche Dimensionen bei relativ geringen Graden der Myopie annimmt. Hier liegt die Vererbung differenter 
Elemente auf der Hand; und ich würde nicht säumen, das von mir in dieser Richtung berejts gesammelte Material von dem oben gezeichneten Gesichtspunkt aus zu analysiren, wenn nicht gerade in neuester Zeit die Myopie-Frage, durch den Nachweis des Accommodations-Krampfes als weit verbreiteten ätiologischen Momentes, in so lebhaften Fluss gerathen wäre, dass wir erst eine Klärung der widerstreitenden Anschauungen abwarten müssen, wollen wir anders ein fruchtbares Resultat von einer solchen Untersuchung haben.

\section{N a c h t r a $\mathrm{g}$.}

Heute stellte sich mir Wilhelm Br., 19 Jahre alt, der Sohn von Sebastian Br., vor mit der Klage einer retinalen Asthenopie: er fühlt sich sehr leicht geblendet und dadurch unfähig zu jeder anhaltenden Arbeit; hiermit im Zusammenhange klagt er über häufige Kopfschmerzen, Herzklopfen, Brustbeklemmung und ähnliche Symptome. Die Untersuchung seiner Augen ergiebt Sehschärfe, Brechzustand, Accommodation und Muskelgleichgewicht völlig normal; ophthalmoskopisch: die Netzhautvenen, und besonders die kleineren, etwas stärker geschlangelt und praller gefült, als normal. In den Lungen ist nichts Abnormes zu finden; das. Herz, dessen Spitzenstoss im 5. Intercostalraume etwa 1 Zoll jenseits der Mamillarlinie zu fühlen ist, scheint etwas vergrössert zu sein, Radialarterien eng und gespannt. Der junge Mann ist für sein Alter und seine Grösse ausserordentlich zart construirt und zeigt eine ganz exquisite Erregbarkeit seines Gefässsystems. Die Untersuchung des Urins, die um so mehr gebotea war, als er ïber intermittirende Schmerzhaftigkeit der Nierengegend klagte, ergiebt eine Trübung beim Kochen, die auf Săurezusatz zwar zum grössten Theile schwindet, also für Phosphate zu halten ist, jedoch ein leichtes Opalisiren zurücklässt, das weiterer Auflösung widersteht. Bei der Behandlung des nicht gekochten Urins mit Salpetersäure tritt k eine Triibung ein; ebensowenig sind Formelemente nachzuweisen. Vergleichen wir diesen Befund mit dem von Sebastian und Peter, so dürfte die Annalme, wir liätten es hier mit dem Beginne der erörterten Gefässalteration zu thun, nicht eine unbegründete sein. Ich werde nicht unterlassen, über den weiteren Verlauf dieser Beobachtung zur Zeit Nachricht zu geben. 